TERRA. Revista de Desarrollo Local e-ISSN: 2386-9968

Número 8 (2021), 709-714

DOI 10.7203/terra.8.20274

IIDL - Instituto Interuniversitario de Desarrollo Local

\title{
Reseña. Ciudadanía, autoorganización y poder local. Una aproximación desde la teoría de sistemas
}

\section{Stefano Guidara}

Graduado en Ciencias Políticas y de la Administración Pública (Universidad de Valencia, España)

guidarastefano@gmail.com

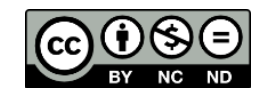

Esta obra se distribuye con la licencia Creative Commons Reconocimiento-NoComercial-SinObraDerivada 4.0 Internacional 


\section{SECCIÓN RESEÑAS}

Reseña. Ciudadanía, autoorganización y poder local. Una aproximación desde la teoría de sistemas

Resumen: El ensayo revisado trata de la exposición y análisis conceptual de la realidad de la autoorganización en sus capacidades, limitaciones y posibilidades futuras. Partiendo de una perspectiva teórica, y pasando por una más práctica, el autor nos expone sus reflexiones para finalmente darnos dos hipótesis de transformación de las sociedades, hacia los sistemas autoorganizativos propuestos.

Palabras clave: perspectiva institucional, sistema social, participación civil, democracia, desarrollo sostenible

Recibido: 21 de enero de 2021

Devuelto para revisión: -

Aceptado: 22 de enero de 2021

\section{Referencia / Citation:}

Guidara, S. (2021). Reseña. Ciudadanía, autoorganización y poder local. Una aproximación desde la teoría de sistemas. TERRA. Revista de Desarrollo Local, (8), 709-714. DOI 10.7203/terra.8.20274 


\title{
Josep Pont Vidal
}

\section{CIUDADANÍA, AUTOORGANIZACIÓN Y PODER LOCAL. UNA APROXIMACIÓN DESDE LA TEORÍA DE SISTEMAS}

\author{
Madrid (España), Catarata, 2019, 192 páginas
}

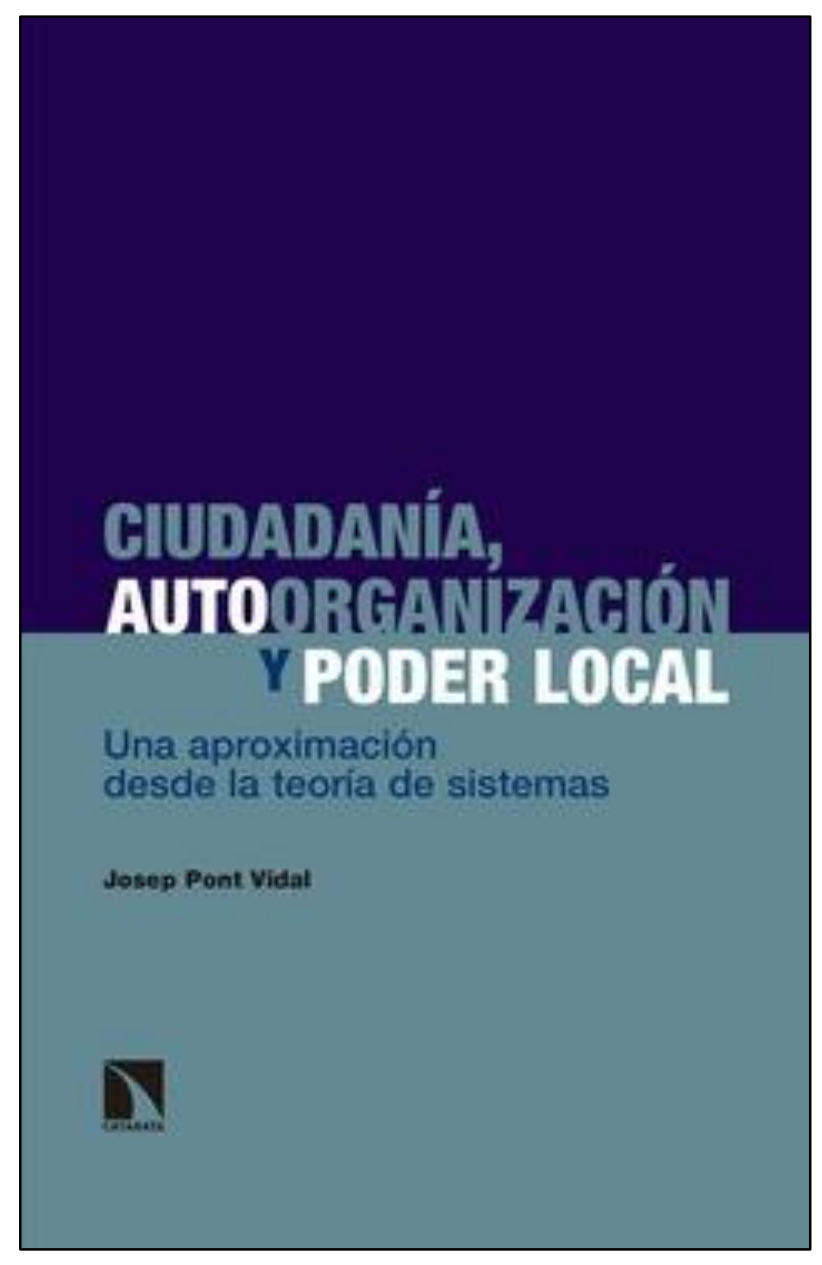

El libro que reseñamos a continuación trata sobre la exposición y el análisis conceptual de la realidad de la autoorganización en sus capacidades, limitaciones y posibilidades futuras. La obra comienza con un amplio y sólido componente teórico, pasando seguidamente por una parte más práctica, en la que el autor nos desgrana su pensamiento para, finalmente, darnos dos hipótesis de transformación de las sociedades hacia los sistemas autoorganizados. A continuación, daremos énfasis sobre los aspectos planteados en cada capítulo.

En el primer capítulo, el autor nos introduce en el marco teórico de su pensamiento. Las sociedades de nuestro planeta se encuentran en un fin de ciclo, ya que, en sus investigaciones, nos relata como las teorías sociológicas y descripciones que hasta la fecha hemos utilizado, van quedándose estancadas y empiezan a mostrar sus limitaciones (y límites). Diversas publicaciones se hacen eco de que estamos en el inicio de una nueva era, el "Antropoceno". En una cita de Manuel de Cózar (2019), esta nueva era se define como "una nueva época geológica, en la cual la humanidad constata su poder para transformar la realidad terrestre a escala global, al tiempo que observa con preocupación las reacciones que está provocando". En estos primeros acordes de la publicación, el autor también hace enfoque en la sociedades policéntricas, que son aquellas más recientes y que mejor explican el funcionamiento de las mismas. Dentro de esas sociedades policéntricas, uno de los centros clave está constituido por las ciudades. En ellas, las administraciones municipales rigen su paso en un contexto de complejidad, diversidad y dinamismo.

El segundo capítulo se inicia con un acercamiento al concepto de "autoorganización". El autor nos habla de las diferentes teorías en torno al término, tanto desde las Ciencias Sociales o Humanas y las Ciencias Ecológicas. Entre las diferentes aproximaciones que hace al término de autoorganización, resulta interesante la diferenciación entre autoorganización política institucional y no institucional, cómo surgen esas formas de organizarse, y los diferentes actores e interacciones que las caracterizan. Con relación a las interacciones, el autor dedica un pasaje en este capítulo a describir cómo estas se relacionan con el concepto en cuestión (autoorganización). En una concepción 
policéntrica, en cualquier sistema organizativo se necesita de interacciones comunicativas entre los diferentes actores que componen este, que pueden derivar en éxito o conflicto. El éxito se dará cuando el destinatario, el poder local, es accesible y acepta la comunicación y el código con el que se comunicará. Esta interacción puede ser explicada desde tres diferentes perspectivas: la conceptual de elección pública, la neomarxista y la institucional.

En la institucional, las reglas del juego o de interacción son las que imponen las instituciones. En la perspectiva marxista y neo-marxista se parte de la base de la premisa del binomio clásico de descripción de la sociedad (explotadores y explotados), y la resultante dialéctica de la lucha de clases y de las consecuencias de la movilización social derivadas de la autoorganización. La perspectiva de la elección pública, derivada de la institucional, nos dice que la elección racional aplicada en los gobiernos locales presentaba una serie de ventajas frente a los habituales gobiernos centralizadores por los siguientes motivos: por un lado, al disponer los municipios de una mayor competencia, se produciría una mayor recaudación de impuestos; y, por otro, puesto que los acuerdos y la toma de decisiones entre pequeños municipios es preferible a un tipo de directriz procedente de una unidad mayor, se reforzaría el poder local. Así, las instituciones urbanas pueden facilitar la coordinación y la cooperación.

El capítulo tercero del libro es el de mayor interés para quien escribe estas líneas, ya que podríamos decir que el autor pasa de una concepción teórica de los planteamientos del libro a una parte más práctica, tangible, haciéndonos ver unos procesos de autoorganización históricos, analizándolos y exponiéndolos a partir de dos posibilidades, la perspectiva institucional (inicio ciudadano con apoyo institucional), y la perspectiva de proyectos surgidos de la gestión promovida por iniciativas ciudadanas sin apoyos institucionales externos.

El primer ejemplo empleado en esta parte más práctica, hace referencia al caso de 'Móstoles en transición'. En el año 2013 se iniciaron las primeras reuniones con diversos colectivos de la ciudad para formular los principios constitutivos de 'Móstoles en transición', que marcaron su posición política y determinarían su evolución en los próximos años. Esta iniciativa tuvo un primer enfoque anticapitalista, aunque no se cerraban a interactuar con diversos actores, aunque siempre manteniendo plena autonomía de decisión respecto a cualquier entidad política. Esta iniciativa promueve democracia plena en su estructura, economía, etc. Además no busca el monopolio de acción, si no que aceptaba que sus premisas pudieran ser aceptadas por otros colectivos. Esto quiere decir que es una especie de "punto de encuentro" de diversos proyectos y colectivos para conseguir una transformación paulatina del sistema social.

El siguiente ejemplo es Holanda y los municipios de Amersfoort y Ámsterdam. La participación y la autoorganización en Holanda tiene una gran tradición. El gobierno ha promovido una iniciativa llamada Haz-democracia, destinada a incentivar y promover las fórmulas de participación ciudadana en los procesos democráticos. En estas ciudades, la tradición democrática y los procesos de autoorganización que sistemáticamente han influido en numerosos procesos cívicos, han conseguido que exista una interacción entre grupos sociales y gobierno en retracción importante.

En Alemania también podemos observar procesos de autoorganización. La participación civil y el compromiso son prácticas extendidas en este país; la clave en esto ha sido la diferenciación entre la sociedad y el Estado en dos esferas diferentes y delimitadas. Siempre ha existido en Alemania un amplio debate sobre el papel de las instituciones representativas y la participación ciudadana. Estas instituciones siguen siendo el lugar 
central para las decisiones que afectan al público en general. Esto permite que, en los procesos de implementación y control, exista una mayor y mejor gobernanza multinivel. En Hamburgo, se organizó el 'Taller Municipal de Hamburgo', para configurar, entre técnicos y ciudadanos, del futuro de la ciudad. Este taller, fue el marco creado en el cual, los diferentes actores pudieron interactuar y diseñar los proyectos urbanos. Como práctica significativa se puede destacar "plan de futuro para Altona" que inició un proceso de participación integral y los planes informales de desarrollo de diversos distritos. En contraposición a estas iniciativas surgidas del poder municipal, podemos destacar iniciativas surgidas desde grupos de participación ciudadanos, como el "NO-BNQ" surgido en el barrio de St. Pauli, famoso por su ideología obrera.

Fuera de Europa, que es la cuna de la democracia y podríamos observar estos procesos de participación y autoorganización como una evolución de la misma a lo largo de los años, también encontramos este tipo de procesos en países con menor tradición democrática. En América Latina tenemos dos ejemplos prácticos en los que observamos procesos de autoorganización y participación multinivel. Diversas instituciones han dejado patentes los problemas financiero-burocráticos y de deuda pública. Para solucionar esto, el gobierno de Michel Temer propuso una serie de reformas que fueron consideradas arbitrarias por la sociedad. Para involucrar a la población se desarrollaron planes de voluntariado para afianzar las políticas, dentro del Programa Nacional de Voluntariado, uniendo la iniciativa gubernamental, la sociedad civil y el sector empresarial.

En la zona amazónica, complicada de delimitar, sin embargo, podemos distinguir procesos de autoorganización en torno a comunidades de explotación de recursos, como la pesquera o la forestal. Estas comunidades forman grupos de gestión comunes, auténticos ejemplos de autoorganización, que forman una identidad política totalmente diferenciable de los poderes públicos gubernamentales.

En el cuarto capítulo, el autor nos habla de varios temas, poniendo énfasis en la lógica sistémica autorreferencial de la administración pública municipal, como subsistema vinculado al aparato estatal, de gobierno y legislativo o de la cooperación y el comunitarismo. Sin embargo, hemos encontrado especialmente interesantes los pasajes en los que el autor nos habla sobre los procesos que nos llevan a una nueva coordinación democracia - mercado - desarrollo sostenible. Esta aproximación es necesaria para responder positivamente a la entrada del Antropoceno. El autor destaca tres nociones centrales: la autoorganización, la cogestión y coprodución de servicios, y la participación.

La autoorganización responde a relaciones de poder de diversas índoles, económicas, políticas, etc. Para lograr la cogestión, se ha de conseguir que la población asuma una serie de responsabilidades y que esté preparada para administrarse. Otro aspecto a recalcar, que sintetiza las posibilidades de unión de entre la autoorganización y el autogobierno es la cogobernanza, una suerte de gobierno horizontal. En su aplicación, los actores se gobiernan, cooperan, y se comunican sin que haya un actor dominante. La participación es la capacidad de expresar decisiones que afectan la vida individual y colectiva.

Para finalizar el libro, se nos proponen diversos párrafos sobre algunas matrices que el autor considera importantes ante la necesidad de un nuevo proceso de orientación, que sobrepase la jerarquía establecida por la acción del Gobierno. Se entiende por superada la acción determinada por el binomio tradicional Mercado - Democracia, y añade el concepto Desarrollo Humano Sostenible como parte indispensable del sistema, 
fundamentado en dos supuestos: i) las prácticas de cogobernanza y autogobierno, y ii) el análisis de la gobernanza con capacidad de englobar los planos teóricos y metodológicos.

Sin embargo, ¿cómo llegar a estos nuevos sistemas planteados por el autor? Se nos ofrecen dos hipótesis: por un lado, el círculo adaptativo heurístico adaptado, poniendo como fases del proceso de autoorganización la innovación, el aprendizaje, la autoorganización y la gestión; y, por otro lado, el sentido y autoorganización, donde, mediante el sentido, la conciencia, la experiencia y la perspectiva holística, la sociedad cambia y se transforma. Sin duda, un panorama lleno de retos en el que demostrar la validez, o no, de ambas opciones.

Stefano Guidara

Graduado en Ciencias Políticas y de la Administración Pública (Universidad de Valencia) 